\title{
Journal of Autism and Developmental Disorders \\ Executive Functions and symptom severity in an Italian sample of intellectually able preschoolers with Autism Spectrum Disorder \\ --Manuscript Draft--
}

Manuscript Number:

Full Title:

Article Type:

Keywords:

Corresponding Author:

Corresponding Author Secondary

Information:

Corresponding Author's Institution:

Ospedale Pediatrico Bambino Gesu

Corresponding Author's Secondary

Institution:

First Author:

First Author Secondary Information:

Order of Authors:

Giovanni Valeri

Laura Casula

Eleonora Napoli

Paolo Stievano

Barbara Trimarco

Stefano Vicari

Teresa Gloria Scalisi

Order of Authors Secondary Information:

Funding Information:

\section{Abstract:}

Response to Reviewers:
A novel battery (BAFE; Valeri et al., 2015) was used in order to assess three Executive Function (EF) abilities (working memory, inhibition and shifting) in a sample of 27 intellectually able preschoolers with Autism Spectrum Disorders (ASD) compared with 27 typically developing children matched on age and nonverbal IQ.

Differences in EF skills were analyzed in participants with distinct ASD symptom severity. Children with ASD performed worse than typical controls on both set-shifting and inhibition, but not on visuo-spatial working memory. Additionally, children with more severe ASD symptoms showed a worse performance on inhibition than children with milder symptoms. These results confirm the presence of EF deficits and highlight a link between ASD symptoms and EF impairments in preschool age.

\section{COMMENTS TO THE AUTHOR:}

> Reviewer \#1: In this study the authors investigated the core executive functions (EF) working memory, inhibition, and shifting using the BAFE test battery in 27 highfunctioning preschoolers with ASD and 27 age and nonverbal IQ matched typically developing children. Differences in these three core EFs were also assessed across participants with distinct ASD symptom severity. Results showed that children with ASD performed worse on the BAFE subtests measuring inhibition and shifting than typically developing children whereas performance on the BAFE subtest measuring working memory was not affected. Furthermore, children with more severe ASD symptomatology showed worse performance on the BAFE inhibition subtest than 
children with milder symptomatology. The authors suggest that these results confirm the presence of EF deficits in preschoolers with ASD and highlight a link between ASD symptomatology and EF.

The authors need to be complimented for their efforts in collecting difficult to obtain data from such a young group of children. In general, this is a concise and well-written paper, methods are sound, results are nicely presented, and the findings are discussed in a clear manner. However, before recommending the paper for publication I have a couple of suggestions for improvement. These are listed below in the order that they appear in the paper.

Introduction

In my opinion the rationale and hypotheses for the study are expressed in a clear way, however I think that the introduction misses some important information on the (early) development of executive functions and the differences in two vs. three factor structure between executive functions in (early) childhood and adulthood (see for instance Best \& Miller, 2010; Lee, Bull, \& Ho, 2013). I believe the authors should address these differences in the Introduction and link this back to the obtained results in the Discussion.

\section{AUTHORS' RESPONSE}

Thank you for this observation, we rewrote most of the introduction according to the Referees' requests.

$>$ Results

Page 7, line 56/57. I cannot follow the sentence: "In order to facilitate the interpretation of the results ...., even the Spin the Pot scores were reflected". Can the authors rewrite this sentence and/or explain what is meant by this statement?

\section{AUTHORS' RESPONSE}

Unfortunately, we wrongly treated the Spin the Pot scores as they were correct responses and after we reflected these scores to obtain error scores. Now we have applied the right procedure, as described at the end of Materials section (page 7, lines 36-40):

"An error score is calculated, by subtracting the maximum correct score (8) from the number of attempts the child makes to find the objects ( $\max 15)$. The error score ranges between 0 and $7 . "$

We then remade all analyses (but the results did not change) and corrected both the values reported in the tables and the discriminant analysis indexes reported in the manuscript.

$>$ Page 9 , line $1 / 2$. Here it is stated that children with a CSS score between 4 and 5 were classified as "less severe ASD". However, a bit further down the sentence (and in Table 4) the mean score of this group is 3.85 . Should this read "between 3 and 5 " or how is a mean score of 3.85 possible?

\section{AUTHORS' RESPONSE}

The CSS range for less severe ASD was between 2 and 5; "4" was a misprint. The mistake was corrected in the manuscript.

$>$ Table 1. It would be informative if Table 1 also included an estimate of verbal-IQ

\section{AUTHORS' RESPONSE}

Unfortunately a measure of Verbal IQ was not administered during the assessment, the IQ was measured with Leiter-R. We added this point to the limitation section.

$>$ Table 3. It seems that the Spin the Pots results are missing from this Table?

\section{AUTHORS' RESPONSE}

The Spin the Pots results were added in Table 3. 
> Reviewer \#2: Thank you for the opportunity to review this paper investigating executive functioning in children with autism spectrum disorder (ASD) without intellectual disability and typically developing controls.

While the authors do a good job providing a rationale for the need to examine executive functioning in preschoolers with ASD, they do a less compelling job reviewing the literature on executive functioning in preschoolers - for example, there are several studies at this point that indicate the executive function in preschoolers is best described by a single factor, e.g., (Lee, Bull, \& Ho, 2013) and thus it is potentially not appropriate to examine the different tasks separately to address this question.

\section{AUTHORS' RESPONSE}

Thank you for this observation, it is certainly important to describe also the single factor framework

to be thorough. We added a paragraph to the introduction where we describe the main frameworks adopted in literature to analyze EF in children.

$>$ The authors also do not make clear whether the literature they cite investigated structured objective tasks or rating scale measures, which do not assess the same thing (Toplak, West, \& Stanovich, 2013).

\section{AUTHORS' RESPONSE}

Most of the studies we cited were conducted using structured objective tasks, only Smithson and colleagues used indirect rating scale measures. We added a sentence in the manuscript to clarify this point (page 3 , line 30 ).

> Additionally, the authors spend time reviewing the literature regarding the association between executive functioning and ASD symptom domains (repetitive restrictive behaviors and social communication deficits) but then do not examine this question in the paper - instead they analyze ASD severity. It may make better sense, especially as they have ADOS data, to look the symptom domains as opposed to a generic "severity" index, so they can integrate their findings into the literature.

\section{AUTHORS' RESPONSE}

The review of literature regarding the association between EF and ASD symptoms is relevant to our research question, as the ASD severity score we used combines the scores of both ASD core domains (CRR and social/interaction deficit), even though it does not consider each domain separately. As added in the manuscript (page 6, lines 20-26) "We chose a comprehensive symptom severity score (CSS), as it can be used to compare ASD symptom severity across individuals of different developmental levels (Gotham et al. 2009), similar to the children with ASD in our sample, who were administered different modules (module 2 or 3 ) of ADOS-G according to their language level."

>Relatedly, in the discussion it is much clearer what the literature shows (e.g., all previous studies with intellectually able preschoolers with ASD reported cognitive flexibility deficits) - this level of clarity should be reproduced in the introduction.

\section{AUTHORS' RESPONSE}

Thank you for this observation, we rewrote most of the introduction according to the Referees' requests.

>Sadly, little information is provided on the sample itself besides age, sex, and nonverbal IQ. The groups are not matched on sex, and thus the authors should at least demonstrate no sex differences on executive functioning between groups if not including it as a covariate in the model.

\section{AUTHORS' RESPONSE}

We assessed sex differences in the control group and reported the results, as follows (page 8, line 57 - page 9 line 2):

"Since the two groups were not matched on gender, we assessed gender differences in the control group only (13 females and 14 males), in order to check if the performance on the Executive Function tasks could be influenced by this factor. ANOVA results showed that gender differences were non-significant for each of the 
four Executive Function measures."

>It would be important to understand the sample in terms of other characteristics (e.g., socioeconomic status, race, attending preschool or not, comorbidities, etc.)

\section{AUTHORS' RESPONSE}

As requested we added available data on preschool attendance and comorbidities in the participants section (page 5, line 6 and line 20).

$>$ The tasks are well described but it is not clear what dependent variables are derived from them, nor how to interpret the scores. It is not easy to interpret, for example, what a value of 12.78 on the working memory STP task means (Table 2)... if the note is correct ("all values are reflected hence they represent errors instead of correct responses"and the STP involves 15 trials) then I would argue that the preschoolers are performing well below chance levels of performance, and this is not a valid assessment of working memory. On the flipside, I'm not sure how meaningful it is that the groups made an average of 1 error only on the Card Sort task (at least on the transformed data), but without the context of a range to refer to it's not possible to understand the significance of these data. Thus, I find myself struggling to understand how these findings advance our understanding of ASD, next steps for research, or clinical implications.

\section{AUTHORS' RESPONSE}

The score range was added to the description of each test in the "Materials" section. As regards the Spin the Pot scores, unfortunately we wrongly treated these scores as they were correct responses and after we reflected them to obtain error scores. Now we have applied the right procedure, as described at the end of Materials section (page 7, lines 36-40): "An error score is calculated, by subtracting the maximum correct score (8) from the number of attempts the child makes to find the objects (max 15). The error score ranges between 0 and 7 ." We then remade all analyses (but the results did not change) and corrected both the values reported in the tables and the discriminant analysis indexes reported in the manuscript.

Concerning the Cart Sort task, the score range is $0-3$. As reported in the new version of the manuscript (page 7, lines 20-22), in the color game "Two trials are compatible with the rule of the prior shape game and three are incompatible. The score is the number of incompatible correct trials; the range is $0-3$." This explains why a low mean number of errors for this taskis reported in table 2 (1.17 corresponding to a row value of 1.4).

$>$ I defer to reviewers with more statistical expertise but it seems that an independent samples t-test would be just as appropriate as the discriminant analysis, and more understandable to the typical reader of this journal. Otherwise some additional (brief) detail is needed on this procedure and why it was selected.

\section{AUTHORS' RESPONSE}

In the results section (Comparison between ASD and Control groups), we added the following (page 9, lines 10-24: "In order to control for the variance shared by the Executive Function scores and reduce type I error probability (Tabachnick and Fidell, 2013) multivariate (discriminant) analysis was used to compare the performances of ASD and control groups, instead of multiple univariate comparisons. In this analysis Card Sort, Night and Day, Pattern Making Test and Spin The Pots scores were the independent variables (predictors) and group membership (ASD vs control group) was the dependent variable. The significance of the Wilks' Lambda value indicates that the linear combination of the predictors significantly discriminates between groups; the effect size (partial 2) represents the percentage of between-group variance explained by the set of predictors and the Partial Lambda is the "unique" contribution of the predictor to the group discrimination, that is after the contribution of the other predictors has been controlled for."

$>$ Effect size estimates would be helpful, perhaps using Hedges $\mathrm{g}$ to address issues related to small sample size.

AUTHORS' RESPONSE 
We added Hedges $\mathrm{g}$ values in the manuscript for predictors with significant Partial Lambdas in the discriminant analyses.

$>$ Additionally, the paper requires considerable editing for English language and grammatical conventions as well as for APA style. Consultation with a native English speaker or scientific writing consultant may be useful.

AUTHORS' RESPONSE

The paper has now been edited by a native English speaker. 
Running head

ASD and Executive Functions in preschoolers

Title

Executive Functions and symptom severity in an Italian sample of intellectually able preschoolers with Autism Spectrum Disorder

\author{
Giovanni Valeri ${ }^{1}$ \\ Laura Casula ${ }^{1}$ \\ Eleonora Napoli ${ }^{1}$ \\ Paolo Stievano ${ }^{2}$ \\ Barbara Trimarco ${ }^{1}$ \\ Stefano Vicari ${ }^{1}$ \\ Teresa Gloria Scalisi ${ }^{3}$
}

1 Children's Hospital Bambino Gesù - Rome, Italy

2 Azienda Sanitaria Locale ROMA 2 - Rome, Italy

3 Department of Social and Developmental Psychology, Sapienza University of Rome - Rome, Italy

Corresponding author:

Barbara Trimarco

Email: barbara.trimarco@gmail.com

Telephone number: $0039-3474939458$

Address: Ospedale Pediatrico Bambino Gesù - Viale di San Paolo, 15 - 00146 Roma 
Title

\title{
Executive Functions and symptom severity in an Italian sample of intellectually able preschoolers with Autism Spectrum Disorder
}

\begin{abstract}
A novel battery (BAFE; Valeri et al., 2015) was used in order to assess three Executive Function (EF) abilities (working memory, inhibition and shifting) in a sample of 27 intellectually able preschoolers with Autism Spectrum Disorders (ASD) compared with 27 typically developing children matched on age and nonverbal IQ.

Differences in EF skills were analyzed in participants with distinct ASD symptom severity. Children with ASD performed worse than typical controls on both set-shifting and inhibition, but not on visuo-spatial working memory. Additionally, children with more severe ASD symptoms showed a worse performance on inhibition than children with milder symptoms. These results confirm the presence of EF deficits and highlight a link between ASD symptoms and EF impairments in preschool age.
\end{abstract}

Key words: working memory, inhibition, shifting, autism spectrum disorder, preschoolers 


\title{
Executive Functions and symptom severity in an Italian sample of intellectually able preschoolers with Autism
}

\section{Spectrum Disorder}

\begin{abstract}
A novel battery (BAFE; Valeri et al., 2015) was used in order to assess three Executive Function (EF) abilities (working memory, inhibition and shifting) in a sample of 27 intellectually able preschoolers with Autism Spectrum Disorders (ASD) compared with 27 typically developing children matched on age and nonverbal IQ.

Differences in EF skills were analyzed in participants with distinct ASD symptom severity. Children with ASD performed worse than typical controls on both set-shifting and inhibition, but not on visuo-spatial working memory. Additionally, children with more severe ASD symptoms showed a worse performance on inhibition than children with milder symptoms. These results confirm the presence of EF deficits and highlight a link between ASD symptoms and EF impairments in preschool age.
\end{abstract}

Key words: working memory, inhibition, shifting, autism spectrum disorder, preschoolers 


\section{Introduction}

Executive Functions (EFs) are a set of higher cognitive processes able to regulate more automatic processes toward a goal, associated with the prefrontal cortex and with interconnected subcortical systems (Stuss, 1992; Zelazo \& Muller, 2002; Diamond, 2013). The EF construct contributes to the understanding of typical and atypical development, with relevant implications in clinical and educational fields (Diamond, 2016).

There is general agreement that three core EF processes can be identified in adulthood (Diamond, 2013) as was first proposed by Miyake et al. (2000), who differentiated the following EF subdomains: updating (maintaining information in working memory and refreshing it in the presence of new information), inhibition (response inhibition and interference control) and shifting (or switching, the ability to shift attention between two tasks). However, even though results on 8 to 13 year-old children confirmed the three-factor model (Lehto et al. 2003), it is still an open question if this model is appropriate for a child population. In fact Lee, Bull and Ho (2013) showed that many confirmatory factor analytic studies on the EF structure in children failed to find evidence for differentiation into the three Miyake et al.'s factors. Hughes et al. (2010) found that inhibitory control, working memory and planning tapped a single underlying cognitive construct in 4 and 6 year-old children, but the result may have been due to the fact that each domain was assessed using only one task. Conversely, Lee et al. (2013), examining 6 to 15 year-old children, collected different measures for each subdomain considered, i.e. updating (including working memory), inhibition and shifting. It was found that data from the 6 to 13 year-olds conformed to a two-factor model separating updating from a unitary inhibition/shifting factor. For the 15 year-olds, a well-separated three-factor structure emerged. The Lee et al. (2013) results confirm Best \& Miller's (2010) suggestion that the degree of independence of the three Miyake et al.'s FE components changes developmentally. EF impairments have been reported in children and adults with ASD (Hill, 2004). For example, a recent meta-analysis of EFs in ASD (Demetriou et al., 2018) examined 235 studies (from 1980 to 2016), including 6816 participants with ASD and 7265 neurotypical controls. The study confirms a broad executive dysfunction in participants with ASD, relatively stable across development (Demetriou et al., 2018). It is worthy of note that studies with participants below the age of 6 years were excluded from the meta-analysis because the assessment tools presented qualitative differences that rendered the comparisons less valid (Espy, 2004).

Evidence of EF deficits in ASD during the preschool period is less consistent. Some studies, focused on EFs during the early preschool period (below the age of 4 years and 6 months), have not found group differences between children with ASD and mental-age-matched children with typical development (TD) or developmental delay (Griffith, Pennington, Wehner \& Rogers, 1999; Dawson et al., 2002; Yerys et al., 2007). Conversely, some studies involving older preschoolers with ASD showed deficits in shifting and sometimes in other core EFs, such as inhibition and working 
memory (Dawson, Meltzoff, Osterling \& Rinaldi, 1998; Pellicano, 2007; Kimhi, Shoam-Kugelmas, Agam Ben-Artzi, Ben-Moshe, \& Bauminger-Zviely, 2014).

EF impairments were also found in a recent study (Garon, Smith \& Bryson, 2018) involving 34 preschoolers (age range: 36 to 74 months; $M=54.79$ months); the participants were divided in two groups: younger (age < 54 months or mental age $<42$ months) and older (mental age $>42$ months). Simple and complex components of the three EF core domains (inhibition, working memory, cognitive flexibility) were investigated. Results indicated significant differences between the ASD group and the TD group (255 preschoolers with TD, mean chronological age $=42.98$ months) in the three EF core abilities, with more substantial deficits in inhibition and shifting; specific findings were moderated by mental and chronological age.

The study of early development of EFs is of remarkable interest, especially in ASD preschoolers without intellectual impairment, since EF deficits may be related to the intellectual disability more than to ASD per se. Only a few studies investigated EFs in intellectually able preschool children, which is particularly important in order to clarify contrasting data on EF deficits in children with ASD in preschool age. To the best of our knowledge, there are only four EF studies conducted involving preschoolers with ASD without intellectual disability (Pellicano, Maybery, Durkin, \& Maley, 2006; Pellicano, 2007; Smithson et al., 2013; Kimhi et al., 2014). Most of these studies were conducted using structured objective tasks, only Smithson and colleagues (2013) used indirect rating scale measures. The most recent of these studies found that children with ASD (age range 3 to 6 years) demonstrated impairments in cognitive shifting and planning abilities (Kimhi et al., 2014). Smithson and coworkers (2013) found greater impairments in parent-reported real-world EFs (inhibition, shifting, working memory and planning) in children with ASD compared with an age and sex-matched group with TD. Pellicano and colleagues (2006) and Pellicano (2007) reported a worse performance in inhibition, set-shifting and planning in children with ASD compared with a group of matched children with TD. Faja and Dawson (2014) analyzed EFs in a group of slightly older intellectually able children with ASD (6 to7 years) and found impairments in flexibility, but not in verbal working memory, compared with age- and IQ-matched typically developing children.

Therefore, all previous studies involving intellectually able preschoolers with ASD consistently found cognitive flexibility deficits (Pellicano et al., 2006; Pellicano, 2007; Smithson et al., 2013; Kimhi et al., 2014; Faja \& Dawson, 2014) and all studies that evaluated inhibition also found impairments in this function (Pellicano et al., 2006; Pellicano, 2007; Smithson et al., 2013).

Also, the relationship between early development of EFs and the severity of ASD symptoms in preschool age is a relevant issue yet to be explored in depth. A number of studies have also found a relationship between EF skills and the type of ASD symptoms. There is evidence of a relation between executive dysfunctions and restricted, repetitive 
behaviors and interests (RRBIs) of individuals with ASD (Russell, 1997; Hill, 2004; Lopez, Lincoln, Ozonoff \& Lai, 2005; Happé \& Ronald, 2008; Mosconi et al., 2009; Yerys et al., 2009; D’Cruz et al., 2013; Reed, Watts \& Truzoli, 2013). Concerning the relationship between EF and ASD socio-communication symptoms (social interaction and communication), different opinions exist, but several authors have suggested that impaired EFs may also cause some of the social problems found with ASD (Russell, 1997; Hill, 2004). There are very few studies investigating the link between EFs and ASD symptoms in very young children (Pellicano, Maybery, Durkin \& Maley, 2006; Faja \& Dawson, 2014). Pellicano and coworkers (2006) found no relationships between EFs and parent-reported real-world symptoms in children with ASD (age range 4 to 7 years). Faja \& Dawson (2014) replicated these results on intellectually able children with ASD (age range 6 to 7 years): after IQ was controlled for, no difference was found on directly observed ASD symptoms between children with different performances on a card sorting test (a measure of cognitive flexibility). Nevertheless, performance on card sorting distinguished a subgroup with worse social-communication functioning, above and beyond IQ. Moreover, early EF deficits may predict later ASD symptom severity, as shown by a recent longitudinal study (Kenny, Cribb \& Pellicano, 2018).

The literature reviewed shows that EF deficits have been identified in preschool children with ASD, but findings are not always consistent. Moreover, impairments vary in relation to the age range. However, a specific link between EF impairments and severity of ASD symptoms has not yet been clearly identified in the preschool period.

The present study assessed three EF abilities: working memory, inhibition, and shifting, using the recently published BAFE battery (Batteria per l'Assessment delle Funzioni Esecutive in età prescolare; Valeri, Stievano, Ferretti, Mariani \& Pieretti, 2015). Previous research has indicated that the BAFE battery is sensitive to age differences in typically developing children aged 36 to 60 months (Stievano, Ciancaleoni \& Valeri, 2017).

The BAFE battery is based on a concept, credited by the main research on EF topics, that the EF construct is unitary with partially dissociable core components (Miyake et al. 2000; Letho et al., 2003): inhibition, working memory and shifting. BAFE introduces a new clinical practice that adds rigorous measurement tools, with good psychometric properties (dimensionality, reliability, validity), added to clinical observations or questionnaires (Stievano et al., 2017).

Our primary goals were to examine the profile of specific deficits in core EF domains (inhibition, set-shifting, working memory) in preschoolers with ASD without intellectual disability (ID), and to analyze differences in EF skills in children with distinct ASD symptom severity.

Methods

Participants 
The children with ASD recruited for this study were a clinically referred sample of preschoolers, part of a larger pool of

children attending the Child Neuropsychiatry Unit of Rome's Bambino Gesù Children's Hospital, Italy's largest pediatric hospital. The children were diagnosed by a multi-professional team incorporating neuropsychiatrists, psychologists and speech therapists. All participants attended preschool.

Inclusion criteria were:

1) chronological age between 4 and 6 years

2) clinical diagnosis of ASD, according to the criteria of the DSM-5 (APA, 2013),

3) scores above the autism spectrum cut-off on the Autism Diagnostic Interview-Revised - ADI-R (Lord et al., 1994) and/or on the Autism Diagnostic Observation Generic Schedule -ADOS-G (Lord et al., 2000);

4) non-verbal IQ in the normal range (IQ $\geq 85$ )

5) absence of comorbidities.

Twenty-seven children with ASD met the inclusion criteria (24 males and 3 females, mean age 5 years and 2 months). Twenty-seven control group children were selected from the BAFE standardization sample (Valeri, et al., 2015). This sample consists of 141 typically developing children (66 males and 75 females) with a mean age of 5 years, attending kindergarten in an urban area. Control group children were matched to ASD participants for age and non-verbal IQ. The control group was composed of 14 males and 13 females, with a mean age of 5 years and 2 months. Children with certified disabilities or foreign nationality were not selected for this group. Other descriptive statistics of the two groups are reported in Table 1.

\section{Materials}

\section{Cognitive level}

We used the Brief non-verbal intelligence quotient (IQ), from Leiter-R (Roid and Miller, 1997), to evaluate cognitive levels. This abbreviated IQ measure can be used as a rapid estimate of global intellectual level and is based on 4 subtests:

Repeated Patterns: ability to complete the missing part of a model within combinations of repeated figured objects;

Sequential Order: selection of connected stimuli that progress according to a certain logical order;

Figure Ground: identification of masked figures within a complex stimulus;

Form Completion: ability to recognize an entire object from a fragmented set of its parts. 


\begin{abstract}
ASD diagnostic evaluations
Symptoms of ASD were evaluated using the "gold-standard" tests: the Autism Diagnostic Interview- Revised, ADI-R (Lord et al., 1994) and the Autism Diagnostic Observation Schedule-Generic, ADOS (Lord et al., 2000). ADI-R (Lord et al., 1994) is a parent-report semi-structured interview for establishing a clinical diagnosis of autism. ADOS (Lord et al., 2000) is a semi-structured direct assessment of communication, social interaction, and play or imaginative use of materials for individuals suspected of having autism. The calibrated severity score (CSS) index (Gotham et al., 2009) was also calculated. CSS allows the quantification of autism symptoms independently from patients' individual characteristics, such as age and language level. Gotham et al. (2009) mapped raw ADOS totals onto a 10-point severity metric and divided them into three classes: "non-spectrum ADOS class", with severity scores ranging from 1 to 3 ; "ASD class", with a range of 4-5; and "autism class", with a range of 6-10. We chose a comprehensive symptom severity score (CSS), as it can be used to compare ASD symptom severity across individuals of different developmental levels (Gotham et al. 2009), similar to the children with ASD in our sample, who were administered different modules (module 2 or 3 ) of ADOS-G according to their language level.
\end{abstract}

\title{
Executive functioning assessment
}

BAFE, an Italian neuropsychological battery for preschoolers (Valeri et al., 2015), is based on a functional perspective of cognitive domains (Miyake, 2000; Lehto, 2003; Stievano, Valeri, 2013; Stievano, Ciancaleoni, Valeri, 2017). Each subtest is linked to a specific EF subdomain according to the unitary construct of EF with partially dissociable components (Garon, Bryson \& Smith, 2008). Subtests were adapted from tasks selected in EF literature.

Night and Day "Stroop-like day-night task" evaluates inhibition (Carlson, 2005; Gerstadt et al., 1994). The examiner engages the children in conversation about when the sun comes up and when the moon and stars come out. The children are then presented with a white card showing a drawing of a yellow sun and a black card showing a drawing of a white moon and stars, and required to say "night" for the sun card and "day" for the moon/stars card. The score range for correct responses is $0-16$.

The Pattern Making test assesses Attentional Flexibility. This test is an adapted magnet pattern-making task (Frith, 1971; Hughes, 1998) and used as a measure of frontal function (Hughes, 1998; Passler, Isaac \& Hynd, 1985). Children are first shown a sequence of 18 colored circles on a long strip of cards, and asked to name each color in turn. The examiner says "Yes, you see it makes a pattern: blue-blue-red, blue-blue-red, emphasizing the words in a rhythmic way. 
The examiner then instructs each child to try and make exactly the same pattern on a steel rule using a set of red and blue magnets. No feedback is given during the task. The score range for correct responses is from 0 to 6 . This task is related to a Shift type in which conflict occurs at the perceptual not response stage.

Card Sort evaluates set-shifting (Carlson \& Moses, 2001; Frye, Zelazo \& Palfai, 1995; Zelazo, 2003). The children are introduced to two recipe boxes with rectangular slots cut into the top. Target cards (e.g. red bear, blue house) are affixed to the front of the boxes. The examiner presents a series of cards (red and blue bears and houses) and instructs each child to place all the bears in the box with the red bear and to place all the houses in the box with the blue house in the "shape game". After five consecutively correct trials, the experimenter announces that they will stop playing the shape game and now play the "color game". In this case, all the red items should be placed the box with the red bear and all blue items placed in the box with the blue house. Two trials are compatible with the rule of the prior shape game and three are incompatible. The score is the number of incompatible correct trials; the range is from 0 to 3 . This task is related to a Shift type in which conflict occurs at the response stage.

Spin the Pots evaluates Working Memory (Diamond \& Taylor, 1996; Hughes, 1998). In this task each child is asked to place an object (a red ring) in each of the eight different pots arranged on a tray. The tray is then is covered with a scarf and rotated. Each child is required to lift the scarf and choose a pot. Each time a child chooses a baited pot, the object is put into a small reward envelope for the child to keep. This procedure is repeated until eight objects have been found or after 15 trials have been conducted (according to which was completed sooner). An error score is calculated by subtracting the maximum correct score (8) from the number of attempts the child makes to find the objects (max 15). The error score ranges between 0 and 7.

\section{Procedure}

Each participant of the ASD group was assessed in the Child Neuropsychiatry Unit of the Bambino Gesù Children's Hospital in three different sessions (first session: Leiter-R; second session: ADOS-G; third session: BAFE). Children were tested in a small quiet area by clinical psychologists, experienced with ASD children.

Each participant of the control group individually completed the battery in one session, following the standardized format. The children were tested in a small quiet area of their school by two trained examiners (a psychologist and a speech therapist). 
The study protocols were approved by the Ethical Committee of the Bambino Gesù Children's Hospital and the institutional review boards at each school. All parents signed a written informed consent.

The scoring was double-checked by two different psychologists independently.

Data analysis was performed to check item quality, reliability and validity of the instruments.

Results

The characteristics of the ASD group and the control groups (CG) are shown in Table 1. The minimum and maximum non-verbal IQ values of the ASD group were slightly lower than those of the control group, but the difference between groups was not significant $(\mathrm{F}=0.0006 ; \mathrm{df}=1$ and $52 ; \mathrm{p}=0.98)$.

\section{(INSERT TABLE 1 APPROXIMATELY HERE)}

\section{Descriptive statistics of the Executive Function measures in the total sample and data transformation}

Skewness and kurtosis calculated on the executive function measures collapsed across the two groups revealed moderate negative skewness (from -3.19 to -2,02) and high values of kurtosis (from 3.15 to 10.23) for Card Sort, Night and Day and Pattern Making Test scores, suggesting deviation from normality. For each variable the procedure recommended by Tabachnick and Fidell (2013) in the case of negative skewness was followed, reflecting the data (by subtracting each score from the largest one plus 1) and after applying a square root transformation. Thus the transformed scores, representing errors instead of correct responses, were used in further analyses for Card Sort, Night and Day and Pattern Making Test, and the error raw scores were used for the Spin the Pots task. Descriptive statistics of the transformed scores for the total sample $(\mathrm{N}=54)$ are presented in Table 2. After transformation, skewness and kurtosis values were lower than the values obtained for raw data.

\section{(INSERT TABLE 2 APPROXIMATELY HERE)}

\section{Comparison between ASD and Control groups}

Table 3 shows means and standard deviations of the Executive Function measures, calculated separately for the ASD and the control group. Since the two groups were not matched on gender, we assessed gender differences in the control group only (13 females and 14 males), in order to check if the performance on the Executive Function tasks could be 
influenced by this factor. ANOVA results showed that gender differences were non-significant for each of the four Executive Function measures.

\section{(INSERT TABLE 3 APPROXIMATELY HERE)}

In order to control for the variance shared by the Executive Function scores and reduce type I error probability (Tabachnick and Fidell, 2013), multivariate (discriminant) analysis was used to compare the performances of ASD and control groups, instead of multiple univariate comparisons. In this analysis, Card Sort, Night and Day, Pattern Making Test and Spin the Pots scores were the independent variables (predictors) and group membership (ASD vs control group) was the dependent variable. The significance of the Wilks' Lambda value indicates that the linear combination of the predictors significantly discriminates between groups; the effect size (partial $\eta^{2}$ ) represents the percentage of between-group variance explained by the set of predictors and the Partial Lambda is the "unique" contribution of the predictor to the group discrimination, i.e. after the contribution of the other predictors has been controlled for.

Table 3 shows that the standard deviations of the ASD group were generally slightly higher than the standard deviations of the control group, but the discriminant analysis is robust to violation of the assumption of equality of within-group variance-covariance (dispersion) matrices when sample sizes are equal (Tabachnick \& Fidell, 2013).

The set of Executive Function measures significantly discriminated between ASD and Control groups (Wilks' Lambda $\left.=0.82 ; \mathrm{F}_{(4,49)}=2.73 ; \mathrm{p}<0.05\right)$. The effect size (partial $\eta^{2}$ ) was 0.18 thus the set of predictors explained $18 \%$ of the between-group variability. Only Card Sort and Night and Day scores uniquely contributed significant variance to the group differences (Partial Lambda $=0.926$ and 0.925 respectively; $p<0.05$; with effect sizes Hedges $g=0.67$ and 0.69 respectively). Table 3 shows that ASD children performed worse than controls on both tests.

\section{Comparison between children with higher and lower ASD symptom severity}

The ASD group was divided into two subgroups on the basis of the CSS. Children whose CSS was between 2 and 5 were classified as "less severe ASD" (ASD-: $\mu=3.85 \pm 0.9 ; \mathrm{N}=13$, with 4 children having a CSS score of 2 or 3 ) while children whose CSS was 6 or higher were classified as "more severe ASD" (ASD+: $\mu=6.36 \pm 0.74 ; \mathrm{N}=14$ ).

The descriptive statistics of the two subgroups are presented in Table 4. 
ASD- and ASD+ children were compared by means of a discriminant analysis with Age, non-verbal IQ and the four Executive Function measures as independent variables. Even though the sample size of the two groups was small, the statistical power of the discriminant analysis is not drastically reduced if the sample size of the smallest group exceeds the number of predictor variables and sample sizes are not highly unequal (Tabachick \& Fidell, 2013). The set of predictors did not discriminate between ASD- and ASD + children (Wilks' Lambda $=0.63 ; \mathrm{F}_{(6,20)}=1.95 ; \mathrm{p}=0.12$ ) but the unique contribution of the Night and Day score was significant (Partial Lambda $=0.71 ; \mathrm{p}<0.01$; Hedges $g=0.72$ ), with ASD+ children performing worse than ASD- (see Table 4).

\section{Discussion}

This study examined the presence of deficits in EF abilities of inhibition, set-shifting and working memory in 27 preschoolers with ASD, without intellectual disability, compared with 27 typically developing children. The study also analyzed differences in EF skills in children with distinct ASD symptom severity.

The ASD group significantly underperformed the control group on both set-shifting (assessed by Card Sort) and inhibition (assessed by Night and Day), but not on visuo-spatial working memory (assessed by STP). These results were independent of IQ and age differences. This pattern of results challenges the Hughes et al.'s (2010) hypothesis that inhibition and working memory can be considered as a unitary construct in 4 to 6 year-old children. Conversely, our findings are in agreement with Lee et al. (2013) results, which showed that at the age of 6 , inhibition and shifting constitute a single factor separated from updating. Lee et al. (2013) also found that inhibition and shifting became clearly separated from one another (and from updating) only at the age of 15 , as efficiency in executive control is not sufficiently specialized and independent until the mid-adolescent years.

Set shifting deficits are common in preschool children with ASD, especially in older preschoolers (Dawson et al., 1998; Pellicano, 2007; Kimhi et al., 2014), even though some works, focused on younger preschoolers, found opposing results (Griffith et al., 1999; Dawson et al., 2002; Yerys et al., 2007). Also inhibition impairments have often been found in studies involving older preschoolers with ASD (Pellicano, 2007; Garon et al., 2018), with some exceptions in younger preschoolers (Yerys et al., 2007). It is important to underline that all previous studies involving intellectually able preschoolers with ASD consistently found cognitive flexibility deficits (Pellicano et al., 2006; Pellicano, 2007; Smithson et al., 2013; Kimhi et al., 2014; Faja \& Dawson, 2014). All studies that evaluated inhibition also found impairments in this function (Pellicano et al., 2006; Pellicano, 2007; Smithson et al., 2013). 
Visuo-spatial WM did not discriminate ASD from typically developing children, thus suggesting that poor working memory may not be a distinguishing characteristic of ASD, in line with Garon and coworkers' (2018) findings on preschoolers. Similarly, Faja and Dawson (2014) did not find impairments in WM, evaluated through a verbal task in a group of 6 to 7 year old children with ASD without intellectual disability.

Also Griffith et al. (1999) showed that, although preschool participants with ASD scored below mental age expectations, their developmentally delayed control group also performed below mental age expectations on most of their measures, including working memory. WM deficits may therefore be associated with developmental delay, and not specifically to ASD. Indeed, WM deficits have been reported in children with Down syndrome compared with mental age matched controls (Daunhaueret al., 2014). Moreover, working memory measures have been strongly associated with overall intelligence (Alloway \& Alloway, 2013), and deficits are characteristic of other developmental disorders such as ADHD (Barkley, 2012).

An EF profile with impairments in inhibition and shifting, but spared WM, was found in relatives of individuals with ASD (Van Eylen et al., 2017), indicating that these functions may be good candidates for endophenotypes, i.e. markers of a disorder closer to genetics (Gottesman and Gould, 2003). These findings are consistent with ours that shifting and inhibition measures differentiated preschoolers with ASD from typically developing children.

Related to the second goal, one of the most relevant evidences of this study is the difference, within the ASD group, in response inhibition (assessed by Night and Day) between the two subgroups with a different severity of ASD symptoms (assessed by ADOS-CSS). Our study highlights the evidence that, in preschool age, children with more severe ASD symptoms show a worse performance in inhibition than those with milder symptoms. This result confirms the presence of a link between ASD symptoms and EF deficits, not only in older children with ASD (Russell, 1997; Hill, 2004), but also in preschool age. In slightly older children, Faja and Dawson (2014) identified a different link between sociocommunication functioning and EF deficits, mediated by cognitive flexibility: cognitive flexibility distinguished a subgroup with worse social-communication functioning above and beyond IQ. Nevertheless, these authors reported no differences in social and repetitive symptoms of ASD based on cognitive flexibility performance, after controlling for IQ. It is important to underline that Faja and Dawson (2014) did not test inhibition, which might explain the difference between their results and ours. Pellicano and coworkers (2006) also reported no relation between EF and social or repetitive symptoms, but they used parent report to assess core autistic symptoms, whereas our findings are based on directly observed symptoms.

The clinical implications of our results suggest that clinicians working with preschool age children with severe ASD symptoms should evaluate all three core EFs during the diagnostic process, as the inhibitory deficit might influence the 
prognosis, and eventually plan specific interventions focused on inhibition skills. Additionally, longitudinal research shows links between early EF deficits and later ASD symptom severity (Kenny et al., 2018).

One of the main limitations of our study is the relatively small sample of preschoolers with ASD. Since ASD is a heterogeneous disorder, the current sample may not be fully representative of ASD. Furthermore, although sex differences were not found, we had only 3 girls in the ASD group. Future studies should recruit larger samples, including more girls. Additionally, a focus on children with different levels of intellectual abilities will be needed in order to generalize these results to the wider population of preschoolers with ASD. Another limitation consists in the lack of a verbal IQ measure in our analyses, which may have added useful information.

Despite its limitations, this study suggests that a profile with typical skills in visuo-spatial working memory and two areas of weakness (inhibition and cognitive flexibility), if confirmed in future research on preschoolers with ASD without ID, could indicate for these children clinical pathways considering specific strengths and weaknesses.

Compliance with Ethical Standards

Ethical Approval

All procedures performed in studies involving human participants were in accordance with the ethical standards of the institutional and/or national research committee and with the 1964 Helsinki declaration and its later amendments or comparable ethical standards. 


\section{References}

Alloway, T.P., \& Alloway, R.G. (2013). Working memory in development. In T. P. Alloway \& R. G. Alloway (Eds.).Working memory: The connected intelligence (pp. 63-82). New York, NY: Psychology Press.

American Psychiatric Association. (2013). Diagnostic and statistical manual of mental disorders (5th ed.). Washington, DC: Author.

Barkley, R.A. (2012). Executive functions: What they are, how they work, and why they evolved. New York, NY: Guilford Press.

Best, J., \& Miller, P. (2010). A Developmental Perspective on Executive Function. Child Development, 81(6), 16411660.doi: $10.1111 / j .1467-8624.2010 .01499 . x$

Daunhauer, L.A., Fidler, D.J., Hahn, L., Will, E., Lee, N.R., \& Hepburn, S. (2014). Profiles of everyday executive functioning in young children with Down Syndrome. American Journal on Intellectual and Developmental Disabilities, 119,303-318.doi: 10.1352/1944-7558-119.4.303

Dawson, G., Meltzoff, A. N., Osterling, J., \& Rinaldi, J. (1998). Neuropsychological correlates of early symptoms of autism. Child development, 69(5), 1276-1285.

Dawson, G., Munson, J., Estes, A., Osterling, J., McPartland, J., Toth, K. \& Abbott, R. (2002). Neurocognitive function and joint attention ability in young children with autism spectrum disorder versus developmental delay. Child development, 73(2), 345-358.

Demetriou, E.A., Lampit, A., Quintana, D.S., Naismith, S.L., Song, Y.J.C., Pye, J.E., Hickie, I. \& Guastella A.J. (2018) Autism spectrum disorders: a meta-analysis of executive function. Molecular Psychiatry, 23, 1198-1204.

Diamond, A. (2013). Executive functions. Annual review of psychology, 64, 135-168.

Diamond, A. (2016). Why improving and assessing executive functions early in life is critical. In J. A. Griffin, P. McCardle, L. S. Freund, J. A. Griffin, P. McCardle, \& L. S. Freund (Eds.), Executive function in preschool-age children: Integrating measurement, neurodevelopment, and translational research (pp. 11-43). Washington, DC: American Psychological Association. doi:10.1037/14797-002

Espy K.A. (2004). Using developmental, cognitive and neuroscience approaches to understand executive control in young children. Developmental Neuropsychology, 26, 379-384.

Faja, S., \& Dawson, G. (2014). Brief Report: Performance on the Dimensional Change Card Sort and Backward Digit Span by young children with autism without intellectual disability. Child Neuropsychology: A Journal on Normal and Abnormal Development in Childhood and Adolescence, 20(6), 692-699. doi:10.1080/09297049.2013.856395 
Garon, N., Smith, I. M., \& Bryson, S. E. (2018). Early executive dysfunction in ASD: Simple versus complex skills. Autism Research, 11(2), 318-330.

Gotham, K., Pickles, A., \& Lord, C. (2009). Standardizing ADOS scores for a measure of severity in autism spectrum disorders. Journal of Autism and Developmental Disorders, 39(5), 693-705.doi:10.1007/s10803-008-0674-3.

Griffith, E. M., Pennington, B. F., Wehner, E. A., \& Rogers, S. J. (1999). Executive functions in young children with autism. Child development, 70(4), 817-832.

Happé, F., Ronald, A. (2008). The fractionable autism triad: a review of evidence from behavioural, genetic, cognitive and neural research. Neuropsychology Review, 18, 287-304.

Hill E. L. (2004). Evaluating the theory of executive dysfunction in autism. Developmental Review, 24, 189-233

Hill, E. L. (2004). Executive dysfunction in autism. Trends in cognitive sciences, 8(1), 26-32.

Hughes, C., Ensor, R., Wilson, A., \& Graham, A. (2010).Tracking executive function across the transition to school: A latent variable approach. Developmental Neuropsychology,35, 20-36.

Kenny, L., Cribb, S., \&Pellicano, E. (2018). Childhood Executive Function Predicts Later Autistic Features and Adaptive Behavior in Young Autistic People: a 12-Year Prospective Study. Journal of Abnormal Child Psychology.doi: 10.1007/s10802-018-0493-8

Kimhi, Y., Shoam-Kugelmas, D., Ben-Artzi, G. A., Ben-Moshe, I., \& Bauminger-Zviely, N. (2014).Theory of mind and executive function in preschoolers with typical development versus intellectually able preschoolers with autism spectrum disorder. Journal of autism and developmental disorders, 44(9), 2341-2354.

Lehto, J. E., Juujärvi, P., Kooistra, L., \& Pulkkinen, L. (2003), Dimensions of executive functioning: Evidence from children. British Journal of Developmental Psychology, 21: 59-80.doi:10.1348/026151003321164627

Lord, C., Risi, S., Lambrecht, L., Cook, E. H., Leventhal, B. L., DiLavore, P. C., \& Rutter, M. (2000). The Autism Diagnostic Observation Schedule - Generic: A standard measure of social and communication deficits associated with the spectrum of autism. Journal of autism and developmental disorders, 30(3), 205-223.

Lord, C., Rutter, M., \& Couteur, A. (1994). Autism Diagnostic Interview-Revised: A revised version of a diagnostic interview for caregivers of individuals with possible pervasive developmental disorders. Journal of Autism and Developmental Disorders, 24(5), 659-685. doi:10.1007/BF02172145.

McEvoy, R. E., Rogers, S. J., \& Pennington, B. F. (1993). Executive function and social communication deficits in young autistic children. Journal of child psychology and psychiatry, 34(4), 563-578.

Miyake, A., Friedman, N.P., Emerson, M.J., Witzki, A.H., Howerter, A., \& Wager, T.D. (2000). The unity and diversity of executive functions and their contributions to complex "frontal lobe" tasks: A latent variable analysis. Cognitive Psychology, 41, 49-100. 
Pellicano, E. (2007). Links between theory of mind and executive function in young children with autism: Clues to developmental primacy. Developmental psychology, 43(4), 974.

Pellicano, E., Maybery, M., Durkin, K., \& Maley, A. (2006). Multiple cognitive capabilities/deficits in children with an autism spectrum disorder: "Weak" central coherence and its relationship to theory of mind and executive control. Development and Psychopathology, 18(1), 77-98.doi:10.1017/S0954579406060056

Roid, G. (1997). Miller L. Leiter international performance scale-revised. Wood Dale, IL: Stoelting.

Russell, J. (1997). Autism as an executive disorder. Oxford University Press, New York.

Smithson, P.E., Kenworthy, L., Wills, M.C., Jarrett, M., Atmore, K., \& Yerys, B.E. (2013).Real world executive control impairments in preschoolers with autism spectrum disorders. Journal of Autism and Developmental Disorders, 43, 1967-1975.doi:10.1007/s10803-012-1747-x

Stievano, P., Ciancaleoni, M., \& Valeri, G. (2017). Italian executive functions battery for preschoolers (BAFE): working memory, inhibition, set-shifting. Neuropsychological Trends, (22), 25-46.

Stievano, P., \& Valeri, G. (2013). Executive functions in early childhood: Interrelations and structural development of inhibition, set- shifting and working memory. Neuropsychological Trends, 13(1), 27-45.

Stuss, D. T. (1992). Biological and psychological development of executive functions. Brain and cognition, 20(1), 8-23.

Tabachnick, B. G. Fidell, L. S. (2013) Using Multivariate Statistics. Pearson New International Edition. Pearson Education Limited.

Turner, M. (1997).Towards an executive dysfunction account of repetitive behaviour in autism. In J. Russell (Ed.), Autism as an executive disorder (pp. 57-100). New York, NY, US: Oxford University Press.

Valeri G., Stievano P., Ferretti M.L., Mariani E., Pieretti E. (2015) BAFE Batteria per l'Assessment delle Funzioni Esecutive, Firenze: Hogrefe Editore.

Yerys, B. E., Hepburn, S. L., Pennington, B. F., \& Rogers, S. J. (2007). Executive function in preschoolers with autism: Evidence consistent with a secondary deficit. Journal of autism and developmental disorders, 37(6), 1068-1079.

Yerys, B.E., Wallace, G.L., Harrison, B., Celano, M.J., Giedd, J.N., \& Kenworthy, L.E. (2009) Set-shifting in children with autism spectrum disorders: reversal shifting deficits on the intradimensional/extradimensional shift test correlate with repetitive behaviors. Autism, 13, 523-538.

Van Eylen, L., Boets, B., Cosemans, N., Peeters, H., Steyaert, J., Wagemans, J., \& Noens, I. (2017). Executive functioning and local-global visual processing: candidate endophenotypes for autism spectrum disorder? Journal of Child Psychology and Psychiatry, 58(3), 258-269.doi: 10.1111/jcpp.12637.

Zelazo, P. D., \& Müller, U. (2002). Executive function in typical and atypical development. Blackwell handbook. 


\begin{tabular}{|c|c|c|c|c|c|c|c|c|c|c|c|}
\hline & \multirow[b]{2}{*}{$\mathrm{N}$} & \multirow[b]{2}{*}{ M } & \multirow[b]{2}{*}{$\mathrm{F}$} & \multicolumn{3}{|c|}{ Age in months } & \multicolumn{5}{|c|}{ Non-verbal IQ } \\
\hline & & & & Mean & SD & Min & Max & Mean & SD & Min & Max \\
\hline ASD group & 27 & 24 & 3 & 61.63 & 6.39 & 49 & 71 & 116.63 & 12.14 & 97 & 135 \\
\hline Control group & 27 & 14 & 13 & 61.63 & 6.32 & 49 & 70 & 116.56 & 9.98 & 102 & 147 \\
\hline
\end{tabular}

Table 1.Characteristics of the clinical and control groups. 
Mean SD Minimum Maximum Skewness Kurtosis

\begin{tabular}{lcccccc}
\hline CS sqrt & 1.17 & 0.31 & 1.00 & 2.00 & 1.751 & 1.974 \\
\hline ND sqrt & 1.57 & 0.75 & 1.00 & 3.87 & 1.659 & 2.611 \\
\hline PMT sqrt & 1.06 & 0.16 & 1.00 & 1.50 & 1.302 & 2.484 \\
\hline STP & 2.88 & 2.49 & 0.00 & 7.00 & 0.534 & -1.089 \\
\hline
\end{tabular}

Table 2. Descriptive statistics of the Executive Function error scores in the total sample $(\mathrm{N}=54)$.

$C S=$ Card Sort $;$ ND = Night and Day; PMT = Pattern Making Test $;$ STP = Spin The Pots; sqrt = square root. 


\begin{tabular}{lllllllll} 
& CS sqrt & & ND sqrt & \multicolumn{3}{c}{ PMT sqrt } & \multicolumn{2}{c}{ STP } \\
\hline & Mean & SD & Mean & SD & Mean & SD & Mean & SD \\
\hline ASD & 1.27 & 0.35 & 1.82 & 0.93 & 1.09 & 0.19 & 3.18 & 2.61 \\
\hline Control group & 1.07 & 0.22 & 1.32 & 0.40 & 1.03 & 0.12 & 2.59 & 2.37 \\
\hline
\end{tabular}

Table 3. Descriptive statistics of the Executive Function error scores in the clinical and control groups.

$C S=$ Card Sort $;$ ND = Night and Day; PMT = Pattern Making Test $;$ STP = Spin The Pots; sqrt = square root. 


\begin{tabular}{llll} 
& & ASD- & ASD + \\
\cline { 2 - 4 } Sample size & & 13 & 14 \\
\hline Age in months & Mean & 60.15 & 63.00 \\
& SD & 7.70 & 4.76 \\
\hline Non-verbal IQ & Mean & 116.85 & 116.43 \\
& SD & 11.72 & 12.95 \\
\hline CS sqrt & Mean & 1.31 & 1.23 \\
& SD & 0.39 & 0.33 \\
\hline ND sqrt & Mean & 1.48 & 2.14 \\
& SD & 0.62 & 1.07 \\
\hline PMT sqrt & Mean & 1.07 & 1.11 \\
& SD & 0.17 & 0.21 \\
\hline SPT & Mean & 2.69 & 3.64 \\
& SD & 2.34 & 2.89 \\
\hline & & & \\
\hline
\end{tabular}

Table 4. Sample sizes and descriptive statistics for the predictors in the discriminant analysis comparing ASD- and ASD+ groups.

$C S=$ Card Sort $; N D=$ Night and Day; PMT = Pattern Making Test $;$ STP = Spin The Pots; sqrt $=$ square root. Values for these tests represent errors. 


\section{Author note}

Giovanni Valeri, Children's Hospital Bambino Gesù - Rome, Italy

Laura Casula, Children's Hospital Bambino Gesù - Rome, Italy

Eleonora Napoli, Children's Hospital Bambino Gesù - Rome, Italy

Paolo Stievano, Azienda Sanitaria Locale ROMA 2 - Rome, Italy

Barbara Trimarco, Children's Hospital Bambino Gesù - Rome, Italy

Stefano Vicari, Children's Hospital Bambino Gesù - Rome, Italy

Teresa Gloria Scalisi, Department of Social and Developmental Psychology, Sapienza University of Rome - Rome, Italy

Correspondence concerning this article should be addressed to:

Barbara Trimarco

Email: barbara.trimarco@gmail.com

Telephone number: 0039 - 3474939458

Address: Ospedale Pediatrico Bambino Gesù - Viale di San Paolo, 15 - 00146 Roma 